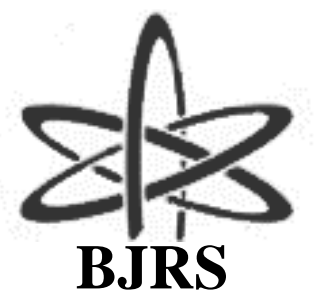

BRAZILIAN JOURNAL

$\mathrm{OF}$

RADIATION SCIENCES

03-02 (2015) 01-12

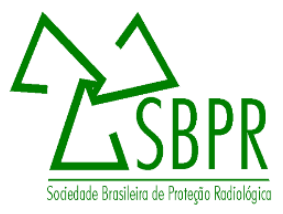

\title{
Individualização das técnicas radiográficas em radiologia computadorizada.
}

\author{
M.E.S. Abrantes ${ }^{\mathrm{a}, \mathrm{b}}$; W. F. Felix ${ }^{\mathrm{c}}$; G. A. P. de Oliveira ${ }^{\mathrm{d}}$; L. G. Stoppa ${ }^{\mathrm{c}}$; \\ O. A. Paiva ${ }^{\text {; }}$ A. H. de Oliveira ${ }^{\text {a }}$ \\ ${ }^{a}$ Departamento de Engenharia Nuclear - DEN, Universidade Federal de Minas Gerais (UFMG), \\ Av. Antônio Carlos, 6627 Campus UFMG, Escola de Engenharia, Bl 3, sala 3011 \\ CEP31270-90, Belo Horizonte - MG, Brasil \\ marcosabrantes2003@yahoo.com.br \\ ${ }^{b}$ Instituto de Pós-Graduação/Feluma - Faculdade Ciências Médicas de Minas Gerais (FCMMG) \\ Pós-graduação em Higiene Ocupacional, Alameda Ezequiel Dias, 275. $2^{\circ}$. andar \\ CEP: 30130-110, Belo Horizonte - MG, Brasil \\ ${ }^{c}$ Unidade Funcional Apoio Diagnóstico e Terapêutica por Imagem - UFADTI \\ Hospital das Clínicas - UFMG - Av. Professor Alfredo Balena, 110. \\ CEP 30130-100, Belo Horizonte - MG, Brasil \\ ${ }^{d}$ Departamento de Anatomia e Imagem - Universidade Federal de Minas Gerais (UFMG)
}

Avenida Alfredo Balena, 190 - sl 7, Campus Saúde.

CEP.: 30130-100, BH-MG, Brasil

\begin{abstract}
RESUMO
Este trabalho tem finalidade de produzir informações para implantação da qualidade de imagens em um setor de radiologia baseado em avaliações prévias das imagens por questionários, de aceitação e qualidade, como etapa de reconhecimento dos parâmetros empregados nas técnicas radiográficas. Após esta investigação os dados levantados foram divididos por grupo masculino, feminino, espessura PA e PF, índice de massa corporal, tipos de biótipos, parâmetros antropofórmicos, avaliação corporal associados às constantes na tensão e filtração adicional. Os resultados apresentam a predominância da constante 35 e 40 com filtração adicional de 0,5 a 1,5 mmAl, tensão para o gênero masculino (PA PF) de 86 a 92 kV e 96 a 112 kV, tensão para o gênero feminino de 85 a 98 kV e 96 a 112 $\mathrm{kV}$, respectivamente. A carga aplicada ao tubo para o gênero masculino (PA e PF) está entre 5 a 10 mA.s e 5 a 16 mA.s e para o gênero feminino (PA e PF) está entre 6,3 a 8 mA.s e 9 a 14 mA.s.
\end{abstract}

Palavras-chave: Qualidade de Imagem; Parâmetros Antropofórmicos; Avaliação Corporal. 


\section{INTRODUÇÃO}

Com o aumento das ferramentas computacionais de produção de imagens, nos sistemas de radiologia computadorizada (CR), para auxiliar no diagnóstico ou até mesmo melhorar o planejamento de terapias que alguns procedimentos e protocolos de qualidade de imagens estão sendo desenvolvidos, fundamentados nas imagens de boa qualidade diagnóstica. Desta forma, tornou-se necessário o conhecimento dos processos de implantação da qualidade de imagens e o quanto elas representam na exatidão em visualizar, medir e gerar informações mais reais possíveis.

A qualidade da radiografia pode ser estimada, subjetivamente, por inspeção direta da imagem clínica para determinadas partes anatômicas de interesse. Neste caso, o médico radiologista, atribui notas às radiografias de acordo com critérios de qualidade da imagem estabelecidos na literatura $[1,2]$.

Resultados de vários países têm evidenciado consideráveis variações, em diferentes instalações e até mesmo em uma mesma instalação para o quesito dosimetria de pacientes e qualidade de imagens no radiodiagnóstico $[3,4,5,6,7,8,9,10,11,12]$.

No Brasil, alguns levantamentos de doses em pacientes e qualidade de imagens realizadas em instalações de São Paulo, Rio de Janeiro, Recife e Belo Horizonte têm servido para traçar um panorama das atuais condições dos serviços de radiodiagnóstico [13, 14, 15].

Os programas de controle de qualidade constituem uma parte essencial na prática radiológica e são eficazes em termos de controle de doses de radiação. Estes programas devem ser fomentados em todas as instalações de radiologia médica e devem abranger parâmetros físicos e técnicos importantes no que diz respeito aos exames radiográficos executados. Serão necessários delimitações nos procedimentos, parâmetros técnicos e valores de referência quanto à exatidão das imagens e doses fornecidas aos pacientes [16].

Diante disto, este trabalho busca demonstrar e relacionar as imagens DICOM, baseado na qualidade de imagens de tórax, com avaliação corporal e parâmetros antropofórmicos dos pacientes levantados, auxiliando assim, na composição das técnicas radiográficas a serem empregadas para radiodiagnóstico e a otimização das doses. 


\section{MATERIAIS E MÉTODOS}

Este trabalho foi dividido em duas etapas, sendo a etapa 1 com objetivo de servir como base e pré seleção das imagens para a etapa 2. Este estudo possui a finalidade de auxiliar a seleção das imagens pelos operadores de raio $\mathrm{X}$ fornecendo subsídios para a etapa 2 como base para implantação da qualidade de imagens associadas as técnicas radiográficas, avaliações corporais e parâmetros antropofórmicos.

Etapa 1 - Para o desenvolvimento deste trabalho foram utilizados valores de constantes associadas às tensões $(\mathrm{kV})$ para produzir variações de penetração dos fótons nos tecidos dos pacientes investigados. Para isto, foram adotadas as constantes 30, 35 e 40 combinadas com as espessuras do tronco nas projeções PA e PF como indicado na equação 1:

$\mathrm{kV}=2$. Espessura do tronco + Valor da constante

Eq. 1

Para a determinação da carga aplicada ao tubo (mA.s) foram utilizadas avaliações visual, tato, conhecimento dos operadores e tamanho do campo, em relação a firmeza dos tecidos do tronco dos pacientes. Os valores da carga aplicada ao tubo em combinação a tensão $(\mathrm{kV})$ já são préajustadas na máquina pelo fabricante, entretanto os valores utilizados neste trabalho estão entre 28 e 4 mA.s. Os valores do índice de exposição ( $\mathrm{LgM})$ gerados pelas técnicas radiográficas foram estipulados, para seleção das imagens, com intervalos de 2,16 a 1,49 e 2,35 a 1,51 para masculino PA e PF e de 2,29 a 1,49 e 2,36 a 1,61 para feminino PA e PF respectivamente. A distância foco écran digital (DFE) foi fixado para todos os casos como $180 \mathrm{~cm}$. As técnicas radiográficas com a utilização das constantes e variações do mA.s foram modificadas em relação ao acréscimo de filtração adicional de alumínio (Al) na máquina ao passo que foram realizados os exames, não possuindo portanto, indicação de pacientes para agregar em um determinado grupo de constantes ou de filtrações adicionais. A máquina de raio $\mathrm{X}$ utilizada foi a de modelo Rad Speed da Shimadzu Corporation que possui filtração total de 3,6 mmAl. Nesta etapa foram utilizadas 72 imagens de pacientes masculinos e 86 imagens de pacientes femininos, para a estrutura de tórax PA e PF. 
Foi elaborado um questionário na etapa 1, para investigar parâmetros de interesse técnico de posicionamento, aplicação das técnicas radiográficas e possíveis contribuições do paciente que podem alterar a qualidade das imagens produzidas. Este questionário foi desenvolvido por dois dos autores Tecnólogos em Radiologia com larga experiência em radiologia de tórax e o autor principal, auxiliados pelas literaturas de referências [17, 18, 19]. Este questionário utiliza apenas respostas "Sim" ou "Não" obtendo respectivamente os valores "dez" e "zero". Para que as imagens desta etapa fossem utilizadas, na etapa 2, utilizou-se o critério de seleção por pontuação as quais tivessem 35 pontos em um total de 50 pontos, para cada projeção.

Tabela 1 - Questionário de investigação das imagens para projeções PA e PF - Etapa 1.

\begin{tabular}{|c|c|}
\hline Projeção PA & Projeção PF \\
\hline $\begin{array}{l}\text { PA1. Densidade óptica: a DO* radiográfica está ade- } \\
\text { quada? }\end{array}$ & $\begin{array}{l}\text { PF1. Técnica de exame: Existe sobreposição das cos- } \\
\text { telas (exame não rodado)? }\end{array}$ \\
\hline $\begin{array}{l}\text { PA 2. Técnica de exame: as articulações esterno clavi- } \\
\text { culares estão igualmente afastadas da linha média da } \\
\text { coluna vertebral? }\end{array}$ & $\begin{array}{l}\text { PF 2. Técnica de exame (tempo longo) ou paciente em } \\
\text { movimento: O exame radiográfico não apresenta mo- } \\
\text { vimento cinético (radiografia tremida/borrada)? }\end{array}$ \\
\hline $\begin{array}{l}\text { PA 3. Técnica de exame ou deficiência do paciente: os } \\
\text { pulmões estão bem inspirados (cheio de ar)? }\end{array}$ & $\begin{array}{l}\text { PF 3. Anatomia: É visualizada a base pulmonar (seios } \\
\text { costofrênicos)? }\end{array}$ \\
\hline $\begin{array}{l}\text { PA 4. Anatomia: Os ápices pulmonares estão incluídos } \\
\text { dentro do campo radiográfico? }\end{array}$ & $\begin{array}{l}\text { PF 4. Anatomia: É possível visualizar a linha de con- } \\
\text { torno do hilo? }\end{array}$ \\
\hline $\begin{array}{l}\text { PA 5. Anatomia: Os seios costofrênicos estão bem } \\
\text { visualizados? }\end{array}$ & PF 5. Anatomia: A região cardíaca é visualizada? \\
\hline
\end{tabular}

Etapa 2 - Para fidelizar a melhor técnica radiográfica e associá-la a imagem de boa qualidade, este trabalho levou em consideração os padrões de biótipos humanos, avaliações corporais e parâmetros antropofórmicos de cada paciente, os quais contribuem na mudança de espectros "filltrados", kerma de saída e densidade ótica das imagens.

Para os critérios investigados de biótipos e parâmetros antropofórmicos, dos pacientes, foram levantados e medidos: idade, altura, peso, espessura do tronco nos sentidos Postero Anterior (PA) e Perfil (PF). Na avaliação corporal foi utilizada uma balança de bioimpedância (Análise por Impedância Bioelétrica - BIA), marca Tanita ${ }^{\circledR}$ modelo IRONMEN BC 1500 de $50 \mathrm{kHz}$ e 0,5 $\mathrm{mA}$ que forneceu as informações de porcentagem de gordura total e do tronco, porcentagem de 
massas magra total, porcentagem total de água no corpo, porcentagem de massa muscular total e do tronco.

Para a balança foi realizado o teste de reprodutibilidade das medidas apresentadas pela BIA em série de 5 medidas com intervalo de 60 segundos entre elas de uma amostra de 9 pacientes escolhidos aleatoriamente. Os itens reproduzidos e medidos foram porcentagem de gordura total e do tronco, porcentagem de massas magra total, porcentagem total de água no corpo, porcentagem de massa muscular total e do tronco. Para calcular o valor da reprodutibilidade foi utilizado a equação 2.

$$
\mathrm{R}(\%)=100 \times \frac{\mathrm{L}_{\mathrm{m} x}-\mathrm{L}_{\min }}{\left(\mathrm{L}_{\mathrm{m} \mathrm{x}}+\mathrm{L}_{\min }\right) / 2}
$$

Onde Lmáx e Lmín são os valores máximo e mínimo encontrados.

Após utilizar as técnicas radiográficas indicadas e descriminadas anteriormente, foram realizadas as divisões dos grupos para a etapa 2 , extraídos da etapa 1, na seguinte forma, sequência e ordem: gênero, espessura do tronco no sentido Postero Anterior para formar o grupo de PA, espessura do tronco no sentido Perfil para formar o grupo de PF, Índice de Massa Corporal (IMC), porcentagem total de água no corpo, porcentagem de massa muscular total e do tronco, porcentagem de massa magra total e porcentagem de gordura total e do tronco, LgM. Para calcular o IMC foi utilizado a equação 3.

$$
\operatorname{IMC}=\frac{P}{H^{2}}
$$

Onde P é o peso e H é a altura em metros do indivíduo.

Na etapa 2 os grupos foram divididos entre 38 e 31 pacientes masculinos com projeções PA e PF respectivamente e 29 pacientes femininos com projeções PA e PF. Desta forma, a etapa 1 obteve um total de 316 imagens e na etapa 2 obteve um total de 127 imagens a serem analisadas. A indicação é que um indivíduo de um determinado grupo de projeção PA necessariamente não estará em um grupo de projeção PF correspondente. Os grupos utilizados para este trabalho tiveram pelo menos 3 imagens representativas das divisões. De acordo com a recomendação do fabrican- 
te Agfa, os valores do LgM ideais para projeções e incidências de tórax PA e PF são 1,8 e 2,0 respectivamente [20], os quais foram adotados nesta pesquisa para serem a base do estudo da avaliação da qualidade de imagens. Porém, nesta etapa 2 utilizou o critérios de seleção das imagens que produziram $\operatorname{LgM}$ entre 1,71 a 1,89 para PA e 1,90 a 2,10 para PF que representam assim, um terço da quantidade de imagens da etapa 1.

Para as visualizações e analises das imagens, nas etapas 1 e 2, foi utilizado o software livre Synedra View Personal 3.

Como ainda não existe um protocolo específico para Radigrafias Computadorizadas (CR) o presente estudo utilizou os princípios do protocolo Europeu [1] para gerar os questionários das tabelas 2.2 e 2.3. O questionário sugerido por VAÑ́́ [21] foi adotado na tabela 2.1. Nstes questionários adotados para a etapa 2 foram acrescentados graduações na pontuação, de acordo com os autores, dos itens investigados, o que não existia nestas duas literaturas. As avaliações dos questionários da etapa 2 foram realizados por dois dos autores médicos, do setor de radiologia da instituição onde foi desenvolvido este trabalho. Nas tabelas 2.1, 2.2 e 2.3 estão apresentados os critérios utilizados nesta etapa 2 , acrescidos das graduações nas pontuações.

Tabela 2.1 - Questionário de investigação das imagens para projeções PA e PF - Etapa 2.

\begin{tabular}{|c|c|}
\hline Projeção PA* & Projeção PF* \\
\hline $\begin{array}{l}\text { PA1. Reprodução dos arcos costais, } 6 \text { anteriormente ou } \\
10 \text { posteriores (executou a inspiracão profunda). }\end{array}$ & $\begin{array}{l}\text { PF1. Reprodução de } 10 \text { corpos vertebrais (executou } \\
\text { inspiracão profunda). }\end{array}$ \\
\hline PA2. A apófise espinhal das vértebras dorsais deve & PF 2. Reprodução sobreposta dos arcos costais posteri- \\
\hline estar equidistante das bordas internas das clavículas. & ores (não girou o tórax). \\
\hline PA3. Reprodução da $7^{a}$ vértebra cervical até a base dos & PF 3. Reprodução da $7^{a}$ vértebra cervical até a base dos \\
\hline $\begin{array}{l}\text { seios de costofrênicos (reprodução de toda cavidade } \\
\text { torácica). }\end{array}$ & $\begin{array}{l}\text { seios costofrênicos posteriores (reprodução da cavida- } \\
\text { de torácica inteira). }\end{array}$ \\
\hline $\begin{array}{l}\text { PA4. Reprodução visual precisa dos vasos periféricos e } \\
\text { ambos os hemidiafraomas em anneia. }\end{array}$ & PF 4. Reprodução visual precisa dos vasos periféricos \\
\hline $\begin{array}{l}\text { PA5. Reprodução visual precisa das bordas da silhueta } \\
\text { cardíaca. }\end{array}$ & $\begin{array}{l}\text { PF 5. Reprodução visual precisa da borda posterior da } \\
\text { aorta e ou do coração. }\end{array}$ \\
\hline $\begin{array}{l}\text { PA6. Visualização dos vasos pulmonares através da } \\
\text { silhueta cardíaca. }\end{array}$ & $\begin{array}{l}\text { PF 6. Visualização dos vasos pulmonares através da } \\
\text { silhueta cardíaca. }\end{array}$ \\
\hline
\end{tabular}


Tabela 2.2 - Questionário de investigação das imagens para projeções PA e PF - Etapa 2.

Projeção PA e PF*

1. Pequenos detalhes nodulares em todo o pulmão, incluindo as áreas retrocardíacas: Alto contraste: diâmetro de $0.7 \mathrm{~mm}$; Baixo contraste: diâmetro de $2 \mathrm{~mm}$.

2. Pormenores lineares e reticulares detectáveis até a periferia dos pulmões: Alto contraste: $0.3 \mathrm{~mm}$ de largura; Baixo contraste: $2 \mathrm{~mm}$ de largura.

*-Avaliar cada critério como 5 = Ótimo; 4 = Bom; 3 = Regular ; 2 = Ruim; 1 = Inaceitável. Qualquer área que não seja visualizada devido a uma condição patológica preencher com a letra $\mathrm{P}(0)$ no quadro pertinente.

Tabela 2.3 - Questionário de investigação das imagens para projeções PA e PF - Etapa 2.

\begin{tabular}{|c|c|}
\hline Projeções PA e PF & Referências para pontuação do questionário \\
\hline 1. Densidade da imagem (enegrecimento): Pulmões & $\begin{array}{l}\text { 1) Densidade da imagem: } 5=\text { Ótima, }-2=\text { Muito ene- } \\
\text { grecido, }-3=\text { Pouco enegrecido. }\end{array}$ \\
\hline 2. Densidade da imagem (enegrecimento): Mediastino & $\begin{array}{l}\text { 2) Densidade da imagem: } 5=\text { Ótima, }-2=\text { Muito ene- } \\
\text { grecido, }-3=\text { Pouco enegrecido. }\end{array}$ \\
\hline 3. Contraste & $\begin{array}{l}\text { 3) Contraste: } 5=\text { Ótimo, }-2=\text { Muito alto, }-3=\text { Muito } \\
\text { baixo. }\end{array}$ \\
\hline 4. Nitidez / Resolução & $\begin{array}{l}\text { 4) Nitidez / Resolução: } 5 \text { = Ótima, } 4 \text { = Boa; } 1 \text { = Inacei- } \\
\text { tável. }\end{array}$ \\
\hline 5. Colimação adequada do feixe & $\begin{array}{l}\text { 5) Colimação adequada do feixe: } 5=\text { Ótima, }-2=\text { Ta- } \\
\text { manho de campo muito grande, }-3=\text { Tamanho de cam- } \\
\text { po muito pequeno. }\end{array}$ \\
\hline 6. Aceitabilidade da imagem & $\begin{array}{l}\text { 6) Aceitabilidade do filme: } 5=\text { Totalmente aceitável, } 4 \\
=\text { Provavelmente aceitável, } 3=\text { Somente aceitável sob } \\
\text { âmbito limitado das condições clínicas, } 1=\text { Inaceitá- } \\
\text { vel. }\end{array}$ \\
\hline
\end{tabular}

\section{RESULTADOS E DISCUSSÕES}

Na etapa 1 os resultados do questionário mostram que 75\% dos critérios adotados entre as projeções (PA3, PA4, PA5, PF1, PF2, PF4, PF5 - masculino e feminino e PF3 feminino), são superiores a $74 \%$ de concordância entre os avaliadores. Para 25\% do questionário (PA1, PA2 - masculino e feminino e PF3 masculino) os critérios adotados de investigação das imagens estão abaixo de 55\% de concordância entre os avaliadores, destacando o PA1 - masculino e feminino que está abaixo de $40 \%$ de concordância. Identificando assim, uma boa relação entre a percepção e acuidade entre os avaliadores.

Na etapa 2 os resultados dos grupos, masculino e feminino, estão apresentados nas tabelas 3 e 4 , como os valores do parâmetro antropofórmico, avaliação corporal e LgM. 
Tabela 3 - Divisão dos grupos masculino com valores do parâmetro antropofórmico, avaliação corporal e $\mathrm{LgM}$.

\begin{tabular}{cccccccccc}
\hline \hline & Espessura do & IMC & Água $(\%)$ & $\begin{array}{c}\text { Massa mus- } \\
\text { cular total } \\
\text { Grupo* }\end{array}$ & $\begin{array}{c}\text { Massa } \\
\text { tronco }(\mathrm{cm})\end{array}$ & $\left(\mathrm{kg} / \mathrm{m}^{2}\right)$ & Ascular do & Massa magra & Gordura \\
total $(\%)$ & Gordura do & total $(\%)$ & tronco $(\%)$ & LgM \\
\hline G3PA2223/2 & $22,6 \pm 0,5 * *$ & $22,03 \pm 1,33$ & $60,15 \pm 3,86$ & $46,99 \pm 6,39$ & $28,06 \pm 7,02$ & $50,74 \pm 5,90$ & $16,67 \pm 4,41$ & $17,81 \pm 5,97$ & $1,83 \pm 0,05$ \\
G5PA2425/2 & $24,7 \pm 0,48$ & $22,83 \pm 2,00$ & $61,63 \pm 4,52$ & $53,26 \pm 4,48$ & $29,63 \pm 2,70$ & $56,09 \pm 4,64$ & $14,78 \pm 4,37$ & $15,5 \pm 5,40$ & $1,82 \pm 0,05$ \\
G5PA2425/4 & $24,79 \pm 0,43$ & $26,90 \pm 1,25$ & $55,64 \pm 5,22$ & $52,81 \pm 2,60$ & $29,29 \pm 2,60$ & $55,60 \pm 5,46$ & $22,88 \pm 6,36$ & $24,74 \pm 7,55$ & $1,85 \pm 0,05$ \\
G7PA2627/2 & $26,11 \pm 0,33$ & $22,56 \pm 1,63$ & $57,14 \pm 5,33$ & $48,8 \pm 4,19$ & $28,22 \pm 2,45$ & $52,72 \pm 5,12$ & $18,39 \pm 6,29$ & $19,97 \pm 7,97$ & $1,82 \pm 0,06$ \\
G7PA2627/4 & $26,63 \pm 0,52$ & $27,22 \pm 1,62$ & $51,33 \pm 4,25$ & $49,6 \pm 5,17$ & $27,79 \pm 2,47$ & $52,29 \pm 5,38$ & $28,23 \pm 5,24$ & $30,94 \pm 5,65$ & $1,78 \pm 0,08$ \\
G7PA2627/5 & $26,2 \pm 0,45$ & $31,45 \pm 0,70$ & $50,64 \pm 1,85$ & $57,44 \pm 9,72$ & $31,28 \pm 4,86$ & $60,45 \pm 10,24$ & $29,86 \pm 2,33$ & $33,1 \pm 3,32$ & $1,78 \pm 0,09$ \\
\hline G3PF3032/2 & $31,17 \pm 0,98$ & $21,13 \pm 1,48$ & $58,15 \pm 2,76$ & $41,65 \pm 4,60$ & $24,15 \pm 2,19$ & $43,9 \pm 4,81$ & $18,2 \pm 4,24$ & $20,6 \pm 4,38$ & $2,06 \pm 0,04$ \\
G5PF3334/2 & $33,75 \pm 0,05$ & $23,18 \pm 1,55$ & $62,43 \pm 2,30$ & $49,4 \pm 3,26$ & $27,17 \pm 1,89$ & $52,03 \pm 3,41$ & $13,93 \pm 2,17$ & $14,27 \pm 2,77$ & $2,04 \pm 0,01$ \\
G5PF3334/4 & $33,67 \pm 0,58$ & $27,79 \pm 1,75$ & $50,9 \pm 4,78$ & $50,4 \pm 5,36$ & $28,2 \pm 2,74$ & $53,09 \pm 5,54$ & $29,0 \pm 5,87$ & $32,7 \pm 6,74$ & $2,0 \pm 0,04$ \\
G7PF3536/2 & $35,4 \pm 0,55$ & $23,81 \pm 0,55$ & $60,9 \pm 8,61$ & $53,18 \pm 3,92$ & $29,58 \pm 1,65$ & $56,03 \pm 4,13$ & $16,55 \pm 9,17$ & $17,3 \pm 11,32$ & $1,98 \pm 0,05$ \\
G7PF3536/4 & $35,67 \pm 0,58$ & $26,59 \pm 0,93$ & $54,2 \pm 0,57$ & $53,85 \pm 8,98$ & $29,95 \pm 3,61$ & $56,75 \pm 9,40$ & $24,0 \pm 0,57$ & $26,7 \pm 0,28$ & $2,03 \pm 0,07$ \\
G9PF3743/4 & $37,8 \pm 0,84$ & $27,20 \pm 1,5$ & $52,45 \pm 5,67$ & $60,05 \pm 8,37$ & $32,83 \pm 4,44$ & $63,18 \pm 8,83$ & $26,28 \pm 7,3$ & $28,58 \pm 7,26$ & $1,96 \pm 0,04$ \\
\hline \hline
\end{tabular}

* - Grupos masculinos PA e PF. ** - Média e desvio padrão.

Tabela 4 - Divisão dos grupos feminino com valores dos parâmetros antropofórmicos, avaliação corporal e LgM.

\begin{tabular}{cccccccccc}
\hline \hline Grupo* & $\begin{array}{c}\text { Espessura do } \\
\text { tronco }(\mathrm{cm})\end{array}$ & $\begin{array}{c}\text { IMC } \\
\left(\mathrm{kg} / \mathrm{m}^{2}\right)\end{array}$ & Água $(\%)$ & $\begin{array}{c}\text { Massa mus- } \\
\text { cular total } \\
(\%)\end{array}$ & $\begin{array}{c}\text { Massa } \\
\text { muscular do } \\
\text { tronco }(\%)\end{array}$ & $\begin{array}{c}\text { Massa magra } \\
\text { total }(\%)\end{array}$ & $\begin{array}{c}\text { Gordura } \\
\text { total }(\%)\end{array}$ & $\begin{array}{c}\text { Gordura do } \\
\text { tronco }(\%)\end{array}$ & LgM \\
\hline G6PA2425/2 & $24,5 \pm 0,52 * *$ & $22,13 \pm 1,77$ & $52,28 \pm 4,19$ & $34,64 \pm 5,88$ & $22,14 \pm 5,63$ & $38,16 \pm 4,05$ & $27,48 \pm 6,35$ & $24,44 \pm 9,13$ & $1,81 \pm 0,17$ \\
G8PA2627/4 & $26,44 \pm 0,51$ & $27,11 \pm 1,21$ & $48,38 \pm 3,78$ & $40,58 \pm 5,16$ & $22,93 \pm 2,65$ & $42,76 \pm 5,42$ & $32,99 \pm 5,14$ & $29,69 \pm 6,21$ & $1,80 \pm 0,05$ \\
G10PA2829/5 & $28,5 \pm 0,52$ & $31,91 \pm 1,43$ & $45,28 \pm 2,95$ & $41,42 \pm 5,43$ & $25,01 \pm 3,38$ & $45,26 \pm 3,18$ & $38,12 \pm 3,98$ & $33,7 \pm 5,78$ & $1,82 \pm 0,05$ \\
\hline G4PF2931/2 & $30,23 \pm 0,73$ & $27,47 \pm 1,45$ & $46,98 \pm 4,11$ & $38,91 \pm 3,28$ & $22,08 \pm 1,82$ & $40,99 \pm 3,47$ & $34,98 \pm 5,84$ & $31,41 \pm 7,81$ & $2,03 \pm 0,01$ \\
G6PF3233/2 & $32,67 \pm 0,5$ & $22,83 \pm 1,57$ & $48,66 \pm 4,42$ & $37,34 \pm 7,25$ & $24,3 \pm 5,92$ & $41,73 \pm 2,76$ & $33,49 \pm 6,17$ & $32,9 \pm 7,93$ & $1,95 \pm 0,04$ \\
G6PF3233/4 & $32,4 \pm 0,5$ & $27,73 \pm 1,35$ & $46,81 \pm 3,62$ & $40,78 \pm 4,71$ & $23,19 \pm 2,44$ & $42,94 \pm 4,93$ & $35,51 \pm 4,87$ & $32,2 \pm 6,11$ & $1,97 \pm 0,04$ \\
G8PF3437/4 & $34,86 \pm 1,21$ & $27,67 \pm 1,25$ & $46,13 \pm 2,61$ & $41,13 \pm 3,65$ & $23,37 \pm 2,08$ & $43,37 \pm 3,82$ & $36,7 \pm 3,73$ & $33,81 \pm 5,28$ & $2,05 \pm 0,02$ \\
\hline *-Grupos femininos PA e PF. ** - Média e desvio padrão. & & & & & & & &
\end{tabular}

Nas tabelas 5 e 6 estão apresentadas as médias das maiores pontuações do grupo, dadas pelos avaliadores médicos, das imagens nos grupos masculino e feminino e as técnicas radiográficas que geraram estas imagens.

Nas tabelas 5 e 6 podem ser observados que as constantes predominantes estão entre 35 e 40, as quais geram maiores valores de tensão aumentando o poder de penetração dos fótons gerados. A filtração utilizada teve predominância para o acréscimo de filtros os quais reduziram os fótons de baixas energias que contribuem no borramento das imagens e aumento das doses nos pacientes. As pontuações nas tabelas 5 e 6 estão acima de $81 \%$, em um total de 70 pontos, indicando que o nível de confiança é significativo, entre as avaliações e as técnicas radiográficas empregadas. 
Tabela 5 - Pontuação dos avaliadores médicos para o grupo masculino e as técnicas radiográficas utilizadas.

\begin{tabular}{|c|c|c|c|c|c|c|c|c|}
\hline \multirow[b]{2}{*}{$\begin{array}{c}\text { Grupo } \\
\text { Masculino* }\end{array}$} & \multirow[b]{2}{*}{$\begin{array}{c}\text { Pontuações dos } \\
\text { avaliadores } \\
(\text { Máxima }=70)\end{array}$} & \multicolumn{7}{|c|}{ Técnicas radiográficas } \\
\hline & & Constante & $\begin{array}{c}\text { Filtração } \\
\text { adicional } \\
(\mathrm{mmAl}) \\
\end{array}$ & $\begin{array}{c}\text { Tensão } \\
(\mathrm{kV})\end{array}$ & $\begin{array}{l}\text { Corrente } \\
(\mathrm{mA})\end{array}$ & $\begin{array}{l}\text { Tempo } \\
\text { (s) }\end{array}$ & $\begin{array}{l}\text { Carga } \\
(\mathrm{mA} . \mathrm{s})\end{array}$ & $\begin{array}{c}\text { Tamanho do } \\
\text { campo }(\mathrm{cmxcm})\end{array}$ \\
\hline G3PA2223/2 & $69,0 \pm 0 * *$ & 40 & 0,5 & 86 & 500 & 0,016 & 8 & $37,5 \times 39$ \\
\hline G5PA2425/2 & $69,5 \pm 0.71$ & 40 & 0 & 90 & 500 & 0,018 & 9 & $40 \times 39$ \\
\hline G5PA2425/4 & $68,0 \pm 2,83$ & 40 & 1 & 90 & 507 & 0,014 & 7,1 & $45 \times 44$ \\
\hline G7PA2627/2 & $68,5 \pm 0,71$ & 40 & 1,5 & 92 & 500 & 0,01 & 5 & $40 \times 43$ \\
\hline G7PA2627/4 & $68,0 \pm 0$ & 35 & 1 & 87 & 500 & 0,018 & 9 & $45,5 \times 38$ \\
\hline G7PA2627/5 & $64,0 \pm 4,24$ & 40 & 1 & 92 & 500 & 0,02 & 10 & $44,5 \times 41,5$ \\
\hline G3PF3032/2 & $63,5 \pm 7,78$ & 40 & 0,5 & 104 & 250 & 0,02 & 5 & $38 \times 45$ \\
\hline G5PF3334/2 & $57,0 \pm 12,73$ & 35 & 0 & 101 & 500 & 0,022 & 11 & $34 \times 42,5$ \\
\hline G5PF3334/4 & $59,5 \pm 7,78$ & 30 & 0 & 96 & 500 & 0,032 & 16 & $32 \times 42,5$ \\
\hline G7PF3536/2 & $64,0 \pm 8,48$ & 40 & 0,5 & 112 & 400 & 0,025 & 10 & $32 \times 39,5$ \\
\hline G7PF3536/4 & $59,5 \pm 13,44$ & 35 & 0,5 & 107 & 400 & 0,04 & 16 & $34 \times 41$ \\
\hline G9PF3743/4 & $61,5 \pm 9,19$ & 35 & 0 & 109 & 391 & 0,032 & 12,5 & $34 \times 43$ \\
\hline
\end{tabular}

* - Grupo masculino PA e PF. ** - Média dos maiores valores das pontuações pelos avaliadores e desvio padrão.

Tabela 6 - Pontuação dos avaliadores médicos para o grupo feminino e as técnicas radiográficas utilizadas.

\begin{tabular}{|c|c|c|c|c|c|c|c|c|}
\hline \multirow[b]{2}{*}{$\begin{array}{c}\text { Grupo } \\
\text { Feminino* }\end{array}$} & \multirow[b]{2}{*}{$\begin{array}{c}\text { Pontuações dos } \\
\text { avaliadores } \\
(\text { Máxima }=70)\end{array}$} & \multicolumn{7}{|c|}{ Técnicas radiográficas } \\
\hline & & Constante & $\begin{array}{l}\text { Filtração } \\
\text { adicional } \\
(\mathrm{mmAl})\end{array}$ & $\begin{array}{c}\text { Tensão } \\
(\mathrm{kV})\end{array}$ & $\begin{array}{l}\text { Corrente } \\
(\mathrm{mA})\end{array}$ & $\begin{array}{l}\text { Tempo } \\
\text { (s) }\end{array}$ & $\begin{array}{l}\text { Carga } \\
\text { (mA.s) }\end{array}$ & $\begin{array}{c}\text { Tamanho do } \\
\text { campo }(\mathrm{cmxcm})\end{array}$ \\
\hline G6PA2425/2 & $66,5 \pm 2,12 * *$ & 35 & 1 & 85 & 525 & 0,012 & 6,3 & $40,5 \times 41$ \\
\hline G8PA2627/4 & $66,0 \pm 1,41$ & 35 & 0,5 & 89 & 500 & 0,016 & 8 & $42 \times 40$ \\
\hline G10PA2829/5 & $62,5 \pm 3,54$ & 40 & 1,5 & 98 & 500 & 0,016 & 8 & $44,5 \times 38,5$ \\
\hline G4PF2931/2 & $67,0 \pm 2,83$ & 35 & 0,5 & 97 & 500 & 0,028 & 14 & $32 \times 39,5$ \\
\hline G6PF3233/2 & $66,5 \pm 2,12$ & 35 & 1 & 99 & 500 & 0,018 & 9 & $30 \times 38,5$ \\
\hline G6PF3233/4 & $68,0 \pm 1,41$ & 30 & 0 & 96 & 500 & 0,028 & 14 & $41 \times 43,5$ \\
\hline G8PF3437/4 & $64,0 \pm 4,24$ & 40 & 1 & 112 & 391 & 0,032 & 12,5 & $35 \times 42,5$ \\
\hline
\end{tabular}

* - Grupo feminino PA e PF. ** - Média dos maiores valores das pontuações pelos avaliadores e desvio padrão.

Na tabela 7 estão apresentados as concordâncias entre os avaliadores da etapa 2 para os critérios 1 e 2 da tabela 2.3 que servirão de comparativo com o critério PA1 da tabela 1.

Tabela 7 - Concordância entre os avaliadores da etapa 2 nas avaliações dos critérios 1 e 2 da tabela 2.3.

\begin{tabular}{ccccc}
\hline \hline \multirow{2}{*}{ Critério } & \multicolumn{4}{c}{ Concordância (\%) } \\
\cline { 2 - 5 } & $\begin{array}{c}\text { PA } \\
\text { masculino }\end{array}$ & $\begin{array}{c}\text { PF } \\
\text { masculino }\end{array}$ & $\begin{array}{c}\text { PA } \\
\text { feminino }\end{array}$ & $\begin{array}{c}\text { PF } \\
\text { feminino }\end{array}$ \\
\hline Densidade da imagem (enegrecimento): Pulmões & 79,41 & 93,91 & 84,62 & 80,0 \\
Densidade da imagem (enegrecimento): Mediastino & 78,95 & 50,32 & 96,55 & 86,21 \\
\hline \hline
\end{tabular}

Os resultados dos valores de reprodutibilidade da balança encontrados estão indicados na tabela 8 , mostrando a confiança nas medidas realizadas. 
Tabela 8 - Medida dos maiores valores gerados pela BIA para cálculo de reprodutibilidade - $R(\%)$.

\begin{tabular}{ccccccc}
\hline $\begin{array}{c}\text { Gordura total } \\
\text { do corpo }\end{array}$ & $\begin{array}{c}\text { Gordura } \\
\text { do tronco }\end{array}$ & Peso & $\begin{array}{c}\text { Água total } \\
\text { no corpo }\end{array}$ & $\begin{array}{c}\text { Massa magra } \\
\text { total no corpo }\end{array}$ & $\begin{array}{c}\text { Músculo total } \\
\text { no corpo }\end{array}$ & $\begin{array}{c}\text { Músculo } \\
\text { do tronco }\end{array}$ \\
\hline 6,76 & 14,29 & 0,23 & 2,05 & 2,39 & 2,51 & 2,87 \\
\hline \hline
\end{tabular}

\section{CONCLUSÕES}

Os critérios adotados na etapa 1 mostram que este questionário pode ser empregado para realizar pré seleção de imagens em uma implantação de qualidade no setor de radiologia assim como, melhoramentos em treinamentos dos operadores de máquinas de raio $\mathrm{X}$ diagnóstico reduzindo tempo e dose de radiação ao paciente.

Na etapa 2 foi satisfatório a criação e graduação nas pontuações dos critérios utilizados, uma vez que não existe um protocolo dedicado para radiografias computadorizas, nem em variações das pontuações. As graduações nas pontuações possibilitaram que os avaliadores médicos tivessem formas de graduar sua opinião em relação a observação e acuidade nas imagens.

As técnicas radiográficas de referência neste trabalho podem ser reproduzidas no setor de radiologia assim como servir de norteador para produzir imagens de boa qualidade, respeitando as configurações das técnicas associadas aos parâmetros antropofórmicos e avaliações corporais dos pacientes.

Para os critérios similares PA1 da tabela 1 (etapa 1) e os critérios 1 e 2 da tabela 2.3 (etapa 2) objetivam informações da quantidade de enegrecimento da imagem, porém não apresentaram semelhanças de concordância entre as etapas investigadas. Porém os autores acreditam que para visualizar os pulmões e mediastino a reprodução de densidade óptica foi suficiente.

O método empregado para qualificar as imagens e produzir informações de base para implantação da qualidade de imagens, em um setor de radiologia, apresentam parâmetros importantes para auxiliar neste aspecto além de criarem suportes para otimização das doses e qualidade diagnóstica. 


\section{AGRADECIMENTO}

À Comissão Nacional de Energia Nuclear (CNEN) por proporcionar bolsa de estudo ao autor principal. À Universidade Federal de Minas Gerais pelo apoio educacional.

\section{REFERÊNCIAS}

1. CEC - COMMISSION OF EUROPEAN COMMUNITIES. European guidelines on quality criteria for diagnostic radiographic images. EUR16260, 1996.

2. CEC - COMMISSION OF EUROPEAN COMMUNITIES. European guidelines on quality criteria for diagnostic radiographic images in paediatrics. EUR16261, 1996.

3. RAMLI, K. et. al. Computed and conventional chest radiography: A comparison of image quality and radiation dose. Australasian Radiology, v. 49, p. 460-466, 2005.

4. CIRAJ, O., MARKOVIC, S., KOSUTIC, D., First results on patient dose measurements from conventional diagnostic radiology procedures in Serbia and Montenegro; Radiation Protection Dosimetry (2005) Vol. 113, No. 3, pp. 330-335, 2005.

5. PAPADIMITRIOU, D., PERRIS, A., MOLFETAS, M.F.G., PANAGIOTAKYS, N., MANETOU, A., TSOUROUFLIS, G., VASSILEVA, J., CHRONOPOULOS, P., KARAPANAGIOTOU, O., KOTTOU, S., Patient dose, image quality and radiographic techniques for common x-ray examinations in two Greek hospitals and comparison with european guidelines; Radiation Protection Dosimetry: Vol. 95, No. 1, pp. 43-48 (2001) Nuclear Technology Publishing, 2001.

6. JOHNOSTON, P., BRENNAN, P.C., Reference dose levels for patients undergoing common diagnostic x-ray examinations in Irish hospitals; Radiation Protection Dosimetry Vol. 95, No. 1, pp. $43-48$ (2001), Nuclear Technology Publishing, 2001.

7. FREITAS, M. B. de; Panorama das Exposições Médicas em Radiologia Convencional no Estado de São Paulo. 2005. 126 f. Tese (Doutorado em Ciências) - Instituto de Física, Universidade de São Paulo, 2005.

8. KOTSUBO, M.T.K. Radiografia de tórax: avaliação da qualidade de imagem, doses e custos. Rio de Janeiro: Universidade Federal do Rio de Janeiro (UFRJ), dissertação de mestrado, 2002.

9. OLIVEIRA, M.L., KHOURY, H. Influência do procedimento radiográfico na dose de entrada na pele de pacientes em raios X pediátricos. Radiologia Brasileira, vol. 36, p.105-109, 2003.

10. OSIBOTE, A. O., et. al. Exposição de pacientes e qualidade da imagem em radiografias de tórax: uma avaliação crítica. Radiologia Brasileira, v.40 (2), p.119-122, 2007.

11. GELEINJNS, J.; SCHULTZE KOOL, L.J.; ZOETELIEF, J.; ZWEERS, D.; BROERSE, J. J. Image quality and dosimetric aspects of chest $\mathrm{x}$ ray examinations: measurements with various types of phantoms. Radiation Protection Dosimetry, v.49, p.83-88, 1993.

12. AZEVEDO, A.C.P., MOHAMADAIN, K.E.M., OSIBOTE, O.A., CUNHA, A.L.L., FILHO, A.P., Estudo Comparativo das Técnicas Radiográficas e Doses entre Brasil e Austrália. Radiol. Bras; 38, n.5: 343-346; 2005.

13. ABRANTES, M.E.S; Dose de radiação em pacientes submetidos a exames de raios $X$ diagnóstico em uma unidade de pronto socorro, 2007. Dissertação (Mestrado em Ciências Técnicas Nucleares) - Universidade Federal de Minas Gerais, 2007.

14. LACERDA, M.A.S.; KHUORY, H.J.; DA SILVA, T.A. ; PEREIRA, M.T. ; ROCHA, W.S. ; AZEVEDO, A.C.P. . Radiation dose to children undergoing chest radiography in public and private pediatric hospitals of Belo Horizonte - Brazil. Radioprotección (Madrid), v. XIII, p. 92-94, 2006.

15. ZENÓBIO, M. A. F.; DA SILVA, T. A. Absorbed doses on patients undergoing tomographic exams for presurgery planning of dental implants. Applied Radiation and Isotopes, v. 65, p. 708-711, 2007.

16. Technical and Physical Parameters for Quality Assurance in Medical Diagnostic Radiology; Tolerances, Limiting Values and Appropriate Measuring Methods, BIR Report 18, 1989. 
17. BONTRAGER, K. L.; Tratado de Técnica Radiográfica e Base Anatômica. $5^{\text {rd }}$ ed. Guanabara Koogan, 2008.

18. BIASOLI, Jr. A.; Técnicas Radiográficas. $1 \mathrm{a}^{\text {rd }}$ ed. Ríbio, 2006.

19. BUSHONG, S. C.; Radiologic science for technologists: physics, biology and protection, St. Louis: Elsevier; Mosby, 638 p., 2004

20. SCHAETZING, R.; Management of pediatric radiation dose using Agfa computed radiography. Pediatr Radiol 34(Suppl 3): S207-S214. DOI 10.1007/s 00247-004-1271-z. Approaches and solutions. 2004.

21. VAÑÓ, E.; GUIBELALDE, E.; MORILLO, A.; ALVAREZ-PEDROSA, C. S.; FERNANDEZ, J. M. Evaluation of the European image quality criteria for chest examintions. The British Journal of Radiology, 68, 1349$1355,1995$. 